International Journal of Network Security \& Its Applications (IJNSA), Vol.3, No.1, January 2011

\title{
COMPARISON PROCESS LONG EXECUTION BETWEEN PQ ALGORTHM AND NEW FUZZY LOGIC ALGORITHM FOR VOIP
}

\author{
Suardinata $^{1}$, Kamalrulnizam bin Abu Bakar ${ }^{2}$ and Nimitr Suanmali ${ }^{3}$ \\ ${ }^{1}$ Department of Computer System and Communication, Faculty of Computer Science \& \\ Information Systems, University Teknologi Malaysia, Johor Bahru, Malaysia \\ STMIK Indonesia Padang, Padang Indonesia \\ suardinata@gmail.com, suardinata@yahoo.com \\ ${ }^{2}$ Department of Computer System and Communication, Faculty of Computer Science \& \\ Information Systems, University Teknologi Malaysia, Johor Bahru, Malaysia \\ kamarulefsksm.utm.my, knizameutm.my \\ ${ }^{3}$ Department of Computer System and Communication, Faculty of Computer Science \& \\ Information Systems, University Teknologi Malaysia, Johor Bahru, Malaysia \\ nimitrs@hotmail.com
}

\begin{abstract}
The transmission of voice over IP networks can generate network congestion due to weak supervision of the traffic incoming packet, queuing and scheduling. This congestion negatively affects the Quality of Service (QoS) such as delay, packet drop and packet loss. Packet delay effects will affect the other QoS such as: unstable voice packet delivery, packet jitter, packet loss and echo. Priority Queuing (PQ) algorithm is a more popular technique used in the VoIP network to reduce delays. In operation, the $P Q$ is to use the method of sorting algorithms, search and route planning to classify packets on the router. Thus, this packet classifying method can result in repetition of the process. And this recursive loop leads to the next queue starved. In this paper, to solving problems, there are three phases namely queuing phase, classifying phase and scheduling phase. The PQ algorithm technique is based on the priority. It will be applied to the fuzzy inference system to classify the queuing incoming packet (voice, video and text); that can reduce recursive loop and starvation. After the incoming packet is classified, the packet will be sent to the packet buffering. In addition, to justify the research objective of the $P Q$ improved algorithm will be compared against the algorithm existing $P Q$, which is found in the literature using metrics such as delay, packets drop and packet losses. This paper described about different execution long process in Priority $(P Q)$ and our algorithm. Our Algorithm is to simplify process execution Algorithm that can cause starvation occurs in $P Q$ algorithm.
\end{abstract}

\section{KEYWORDS}

QoS, VoIP, Fuzzy logic, and Delay

\section{INTRODUCTION}

Currently, VoIP technology has been applied to a Wireless Local Area Network (WLAN) technology sector [16]. However, many problems and issues such as delay, jitter, limited bandwidth, and packet loss (packet drop) occur when communicating, which in turn will affect Quality of Service (QoS) performance. In connection with these problems, the delay is one of the problems that affect the voice quality [12] is will be the main focus of paper.

Some Quality of Service (QoS) technology has been used such as Best Effort Service (BeServ), Differentiated Services (DiffServ), Integrated Service (IntServ), and Multi-Protocol Label Switch (MPLS) [2]. DiffServ and MPLS are two examples of many new models in the network architecture. Even though, the two architectures are almost the same, but DiffServ is more 
International Journal of Network Security \& Its Applications (IJNSA), Vol.3, No.1, January 2011

popular and more knowledgeable [19] and [3]. Furthermore, [5]stated that Diffserv can provide QoS from end to end by establishing guarantee in every routing and switching nodes to perform various types of functions related to QoS metrics (bandwidth, delay or packet loss). DiffServ networks can also achieve its goal by separating the edge (which do complex tasks such as traffic classification, traffic marking and traffic monitoring) and the DiffServ core network. This will further be the focus of the paper. Differentiated service model is designed to achieve the QoS requirements of voice. Some queue scheduling algorithm is also introduced in the differentiated service such as First In First Out / First Come First Serve (FIFO / FCFS), Priority Queuing (PQ), Fair Queuing (FQ), and Round Robin (RR) [14]and Error! Reference source not found.

\section{RELATED WORK}

This paper is to continue the previous paper with entitled "Classifying Incoming Packet Using Fuzzy logic for VoIP)" that will be published in Telkomnika journal in August 2010. In this paper focused on analyzed long execution in PQ algorithm then will be compared with our algorithm.

In several publications [20]; [9]; and [17], reveals that PQ algorithm has better performance than the other queue scheduling algorithm. However, generally PQ algorithm also has some problems that cause inefficiencies in the operation. The PQ algorithm is used sorting, searching [15] and route planning in classifying packets. In classifying packets, some issues such as selection of priority packets still occur recursively (repetition) when the operation, that can cause next queue starvation [18]; [10], [20] and [7]. This is due to incoming packet (in the form of voice, video, and data) are sorted (classified) based on priority. Sorting and searching methods will affect the speed in classifying the packets, while the high speed in the classification may also help to send the packet to the destination node more quickly.

Different priorities of data traffic queues in the network as shown in Figure 1. where the voice is the highest priority, video is a medium priority and high priority data is the lowest priority. As the VoIP network, voice must be serviced first before the video and data.

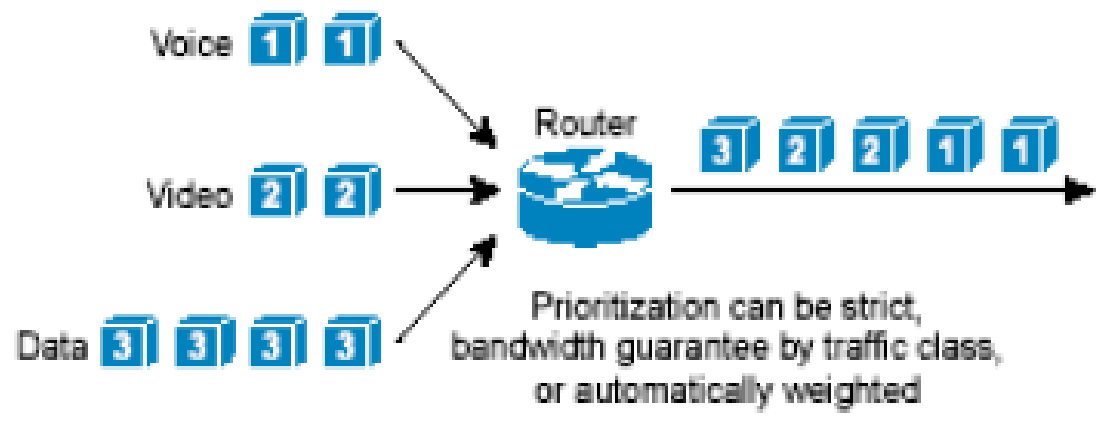

Figure 1. Generic PQ Scheduling algorithms (Source: Kampong J. Mabe, (2005)

In queuing scheduling algorithm that is proposed, the PQ algorithm approach to the incoming packet has been investigated. Some weaknesses in the PQ have been identified as a recursive (repetition) which can lead to lack (starvation) of bandwidth for the next queue, greedy packet buffering is not optimal (inefficient) be used, because of the high priority are always serviced first so that it can cause packet delay. In addition, in this paper a new queue scheduling 
International Journal of Network Security \& Its Applications (IJNSA), Vol.3, No.1, January 2011

algorithm based on PQ algorithm will be improved and applied to a new QoS architecture. Based on the problems and weaknesses that have been identified in the PQ algorithm, then the solution will be proposed in this queue scheduling algorithm. This algorithm is divided into three phases namely queuing classifying, buffering, and scheduling.

To classify an incoming packets, the existing techniques in PQ algorithm will be reduced to three priorities include the High, Medium, and Low. The voice is classified into High, videos are classified into the medium, and the text is classified into Low. New algorithm will be improved by combining existing techniques in PQ algorithms using a fuzzy logic classification system for the packet. The fuzzy logic is simpler and flexible, is able to simplify complex problems and algorithms have been investigated. Fuzzy logic has been used by many interdisciplinary science and applications [22]. In these applications that fuzzy theory can be used to improve the performance of neural networks. For example, fuzzy logic has been used in the simulation of the Microscopic Traffic Network (FLOMITS) model [4] for the car following model. He has compared the traditional model and fuzzy logic models, and revealed that fuzzy logic is more effective and efficient, and it has been used in the proposed model as well. [12] in his paper have also been combined fuzzy logic and genetic algorithms (GA) to solve the access network selection (ANS) problem in heterogeneous wireless networks (HWN). Fuzzy logic has been used by [6] in the queue the packets in the router, where Fuzzy logic is used to classify packets into different priorities based on the packet.

As a justification in this paper, PQ algorithm using sorting methods, search [8]and [15] and planning a route [1] to classify the incoming packet. This algorithm (sorting, and searching) is based on complex mathematical formulas such as bubble sort, insertion sort, heap sort, quick sort, merge sort and Dijkstra's algorithm. Combined techniques in PQ algorithm applied to Fuzzy Logic can classify the incoming packet with intelligent methods. Fuzzy gives priority to each queue based on queue congestion (priority, arrival time and demand). For this paper using the technique in the PQ algorithm and applied to Fuzzy Logic to solve the problem will be proposed.

\section{RESEARCH METHOD}

The research methodology begins with the collection of the literature review that began with the formulation of the problem. In the second step of the experiment test bed was setup to perform real-world scenarios to investigate the QoS parameters and signals that cause latency (delay) in VoIP networks as a process of real-time traffic. After obtaining the results of experiments on a simulation model will be developed in order to reduce the latency (delay) during the conversation so that traffic can be real-time communication continuity without distortion. The main contribution of this paper is to develop an efficient queue scheduling algorithm by using existing techniques in the algorithm PQ. In the last step of performance comparisons will be made to justify the operation and efficiency of queue algorithms developed with QoS the model known currently.

The decision to conduct an experiment test bed comes after an extensive paper of research papers and methods used by other researchers. The idea is to experiment to include VoIP systems, wireless devices and IP phone (soft phone) to obtain real-world results to justify and distinguish the models developed in this paper.

Step-by-step research methodology discussed below and shown in Figure 2: Research Methodology Flowchart. 


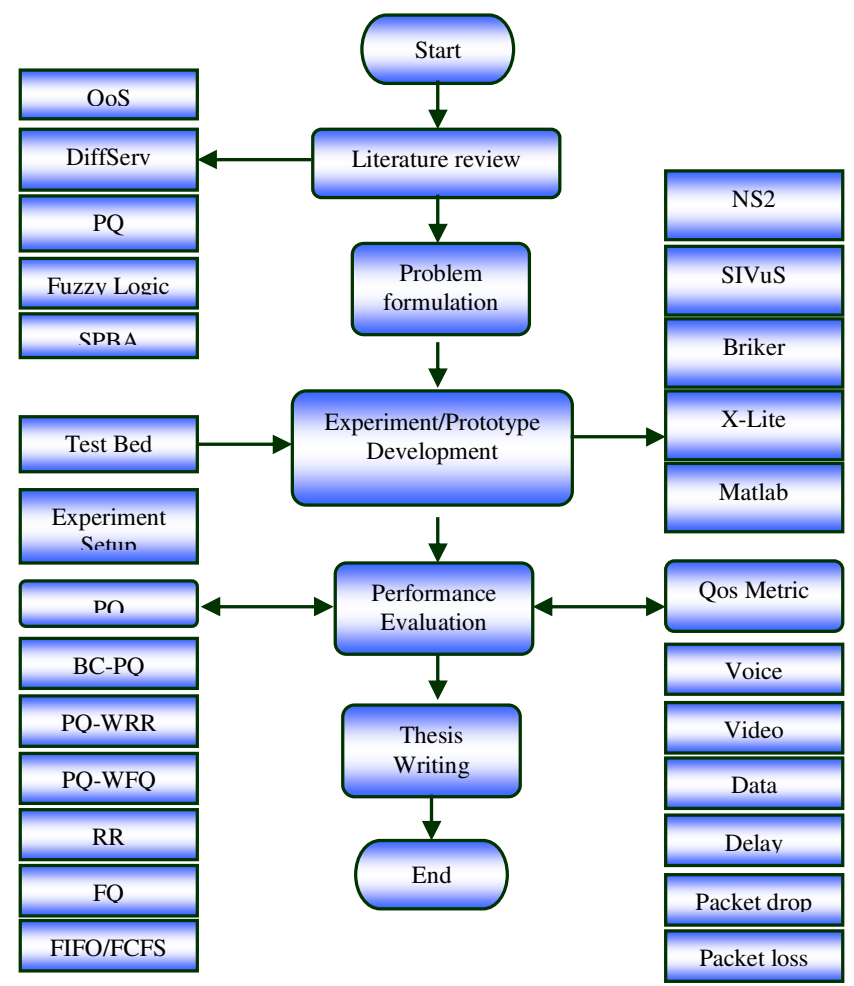

Figure 2. Flowchart of the Research Metodology

There are three phases of the incoming packets data traffic among them is the queuing phase, classification phase, and scheduling phase. First, Phase Queuing is the arrival of incoming packets at random. Incoming packet in the form of voice, video, and data (text) will come randomly and continuously. Voice packets are marked as $\mathrm{H}$ (High), the video packets are marked as M (Medium), and data (text) is marked as L (Low). Second, Classifying phase is where the incoming packets will be selected and classified by priority, arrival and demand. Here, there are three parameters that must be determined to classify traffic based on the data type queue (priority), time of arrival and request packet, while the PQ algorithm is only based on the priority without considering the packet arrival time which can cause a recursive (loop) and starvation. Scheduling phase is where the packets that have been selected (classified) will be scheduled in sequence that started from High, followed by medium and low. In this paper just would be a study about classifying phase. Figure 3 is fuzzy rules based PQ algorithm.

Fuzzy Classifying rules if then based on $P Q$ algorithm

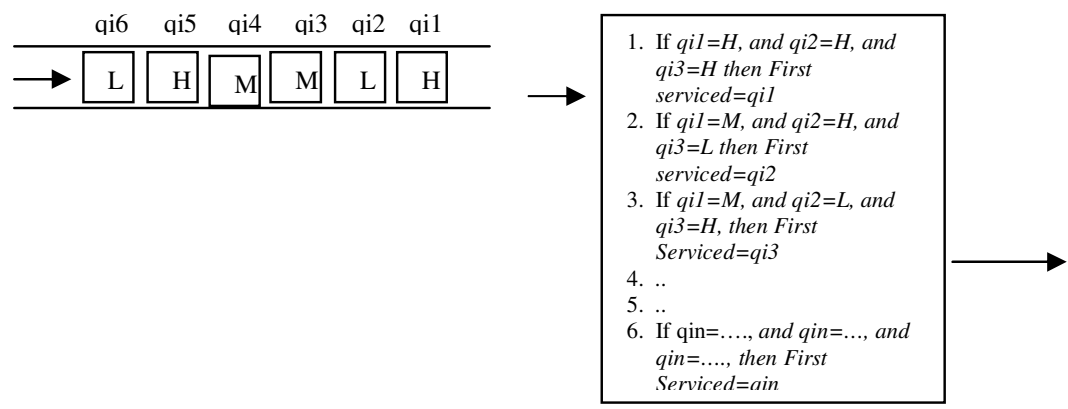


International Journal of Network Security \& Its Applications (IJNSA), Vol.3, No.1, January 2011

Figure 3. Fuzzy process data traffic classifying algorithm

\section{RESULT AND DISCUSSION}

Based on summary of overall information and after PQ algorithm comparison conducted between of them, then a solution queuing scheduling algorithms will be proposed. This solution is consisted of three phases among of are queuing phase, classifying phase and scheduling phase as depicted in Figure 4. Figure 5 and 6 in elaborate detail.

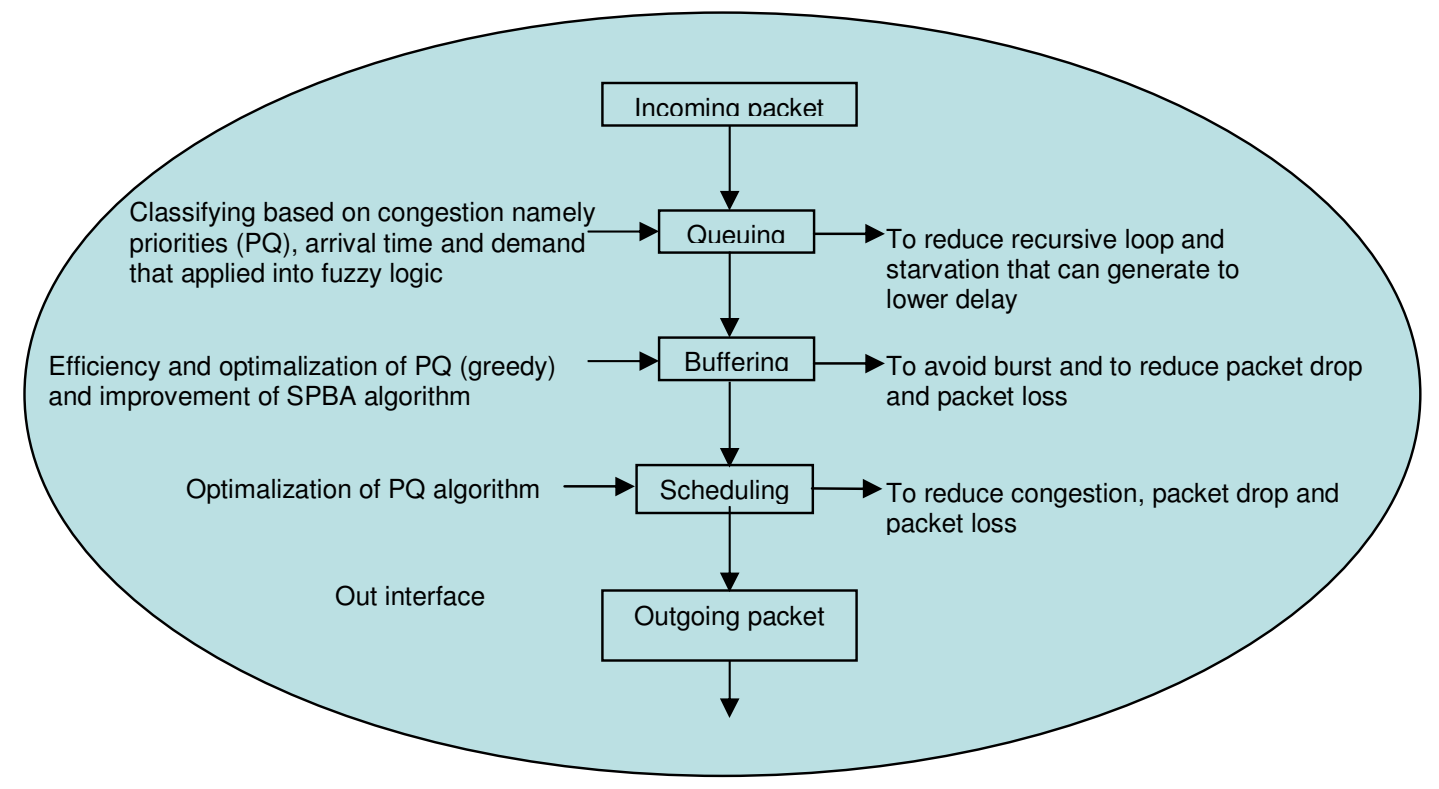

Figure 4. Solution queuing scheduling algorithm

Based on Figure 4. that using existing techniques in PQ algorithms (by priority) and combined with fuzzy logic is a solution that will be offered in a new queue scheduling algorithm. Figure 5. illustrates in detail and drafts a new queue scheduling algorithm. Multimedia data consisting of voice, video, and text (data) are prioritized, depending on the needs. So, the packet classification by PQ algorithm, consisting of four priorities, in this case the priority may be reduced to three priorities, which the voice is the highest priority, video is the medium priority, and data (text) is the lowest priority. After the incoming packet arrives, this packet will be classified into three classes is high for voice, video and Medium to Low to the data (text). Each packet which has been classified by the classifier is shown in Figure 5 .

Incoming Packets

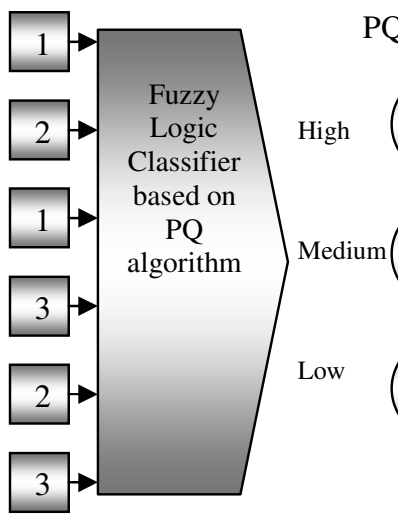

Buffering Packets based on PQ and SPBA algorithm

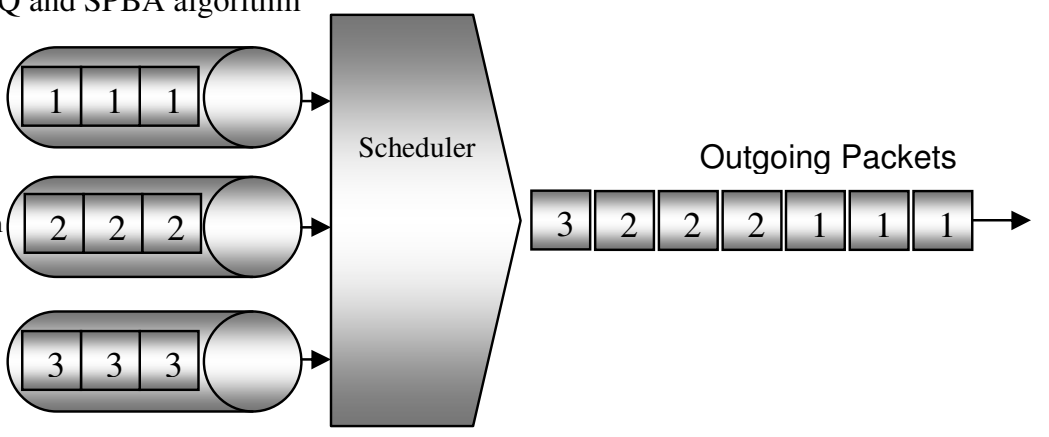


International Journal of Network Security \& Its Applications (IJNSA), Vol.3, No.1, January 2011

Figure 5. Detail new queuing scheduling algorithm

In VoIP network, voice is serviced first, followed by video and data before it is transmitted to other networks. In this paper would combine the existing techniques in the PQ algorithm and Fuzzy logic. Both of these techniques will be applied to the new queuing scheduling algorithm. After the packet is classified, packets transmitted and regulated in the queue buffer to High (voice) placed in the High buffer, to Medium (Video) is placed in the buffer Medium, and Low (data) is placed in a low buffer. In this case, three samples of the queue will be used to make the rules. It aims to facilitate the implementation of the fuzzy logic. New queue scheduling algorithm is expected to be able to manage incoming packet based on priorities.

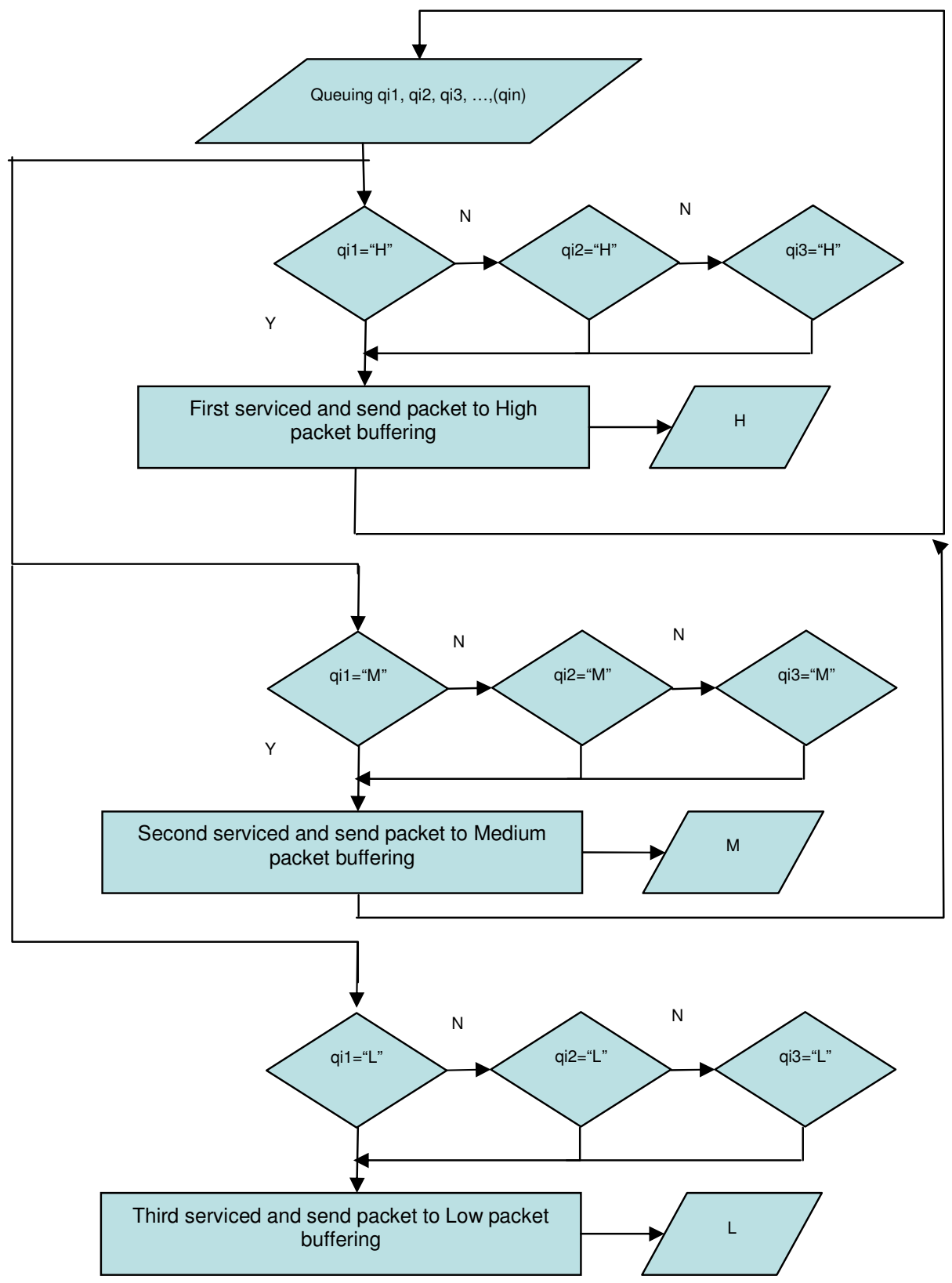

Figure 6. Flowchart new queuing scheduling algorithm 
In this new algorithm (see Figure 6) incoming packet is formed use fuzzy rule namely if then rules. Fuzzy rules consist of input and output. In these case input is qi1, qi2, qi3,.., qin and output is qo. For example is if qi1 is High and qi2 is High and $\cdots$ and qin is High then qo is $q i 1$, etc. And these fuzzy rules are also valid for back queuing continuously as shown in Figure 7.

As shown in Figure 7, there are three phases of the incoming packet data traffic among them is the queuing phase, classifying phase, and scheduling phase. First, Phase Queuing is the arrival of incoming packets at random. Incoming packet in the form of voice, video, and data (text) will come randomly and continuously. Voice packets are marked as $\mathrm{H}$, the video packets are marked as M, and data (text) are marked as L. Second, Classifying phase is where the incoming packets will be selected and classified by priority, arrival and demand. Here, there are three parameters that must be determined to classify traffic based on the data type queue (priority), time of arrival and request packet, while the PQ algorithm is only based on the priority without considering the packet arrival time which can cause a recursive (loop) and starvation. After classifying, the packet will be forwarded to the packet buffering. As in PQ buffer consists of four packets, and also this problem has been described in the introduction, PQ algorithm is using a greedy algorithm. Therefore, in this case simplified into three packets will be proposed that packet buffering should be adjusted to three types of queues High, Medium and Low according to incoming packets as well. Packet buffering algorithm based on PQ and SPBA algorithm. Third, Scheduling phase is where the packets that have been selected (classified) will be scheduled in sequence that started from High, followed by medium and low. As known in the VoIP network, the voice has the highest priority to be serviced first, before video and data. In this algorithm, each packet will be sent based on priority, arrival time, and demand. Based on priority, that means high-priority packets are always transmitted (serviced) first. Based on arrival time is if there are three packets or more of the same after a high priority is to continue next. And on request is that if many high-priority queues after the next will be forwarded.

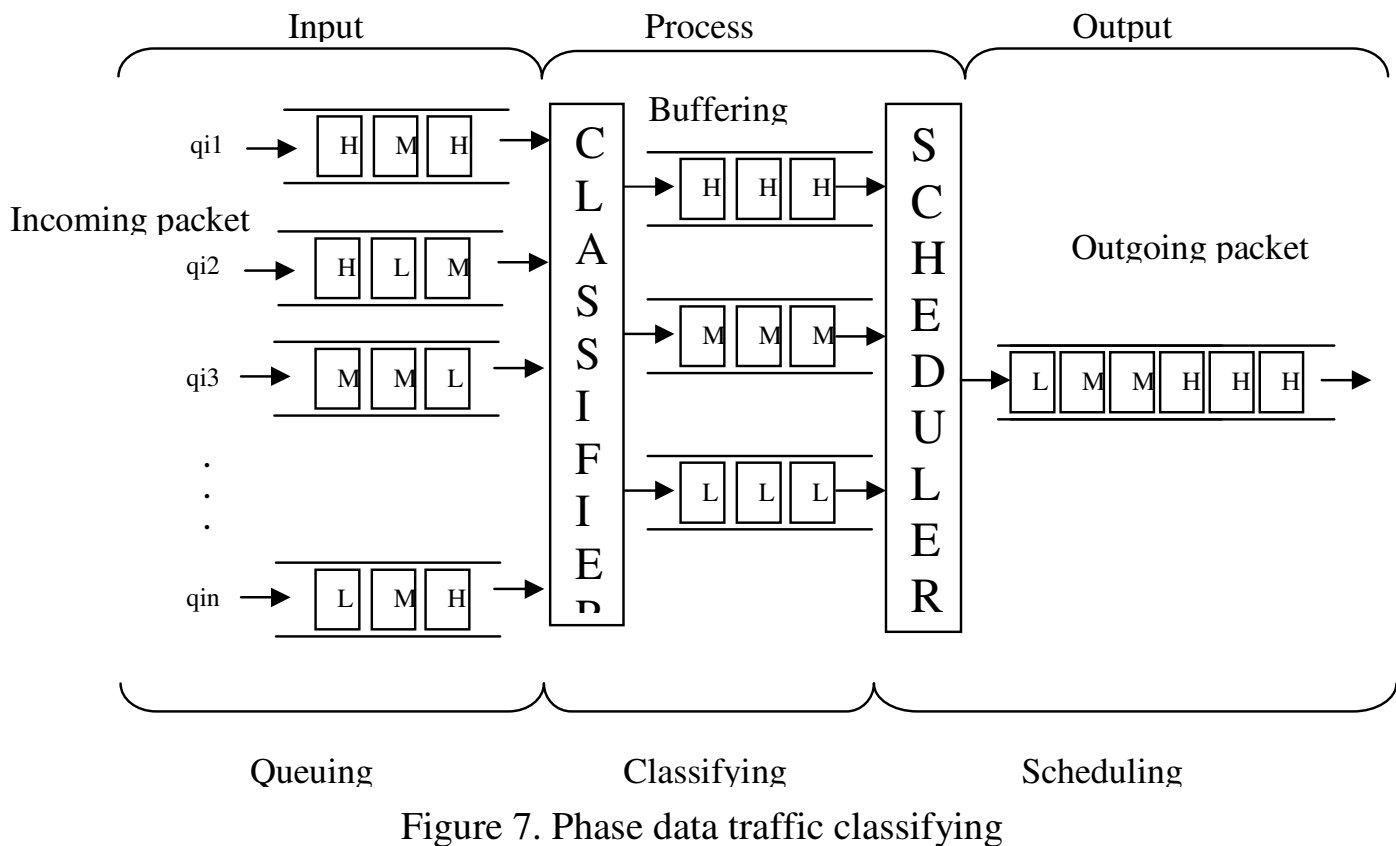


In Figure 8 below has shown an example execution algorithm in PQ algorithm that happening recursively. This algorithm needs many steps that must be executed to sorting priority completed. This algorithm is based on insertion sort method, which has been used in PQ in long time (previous time). In traffic incoming packet on TCP, the long execution will be spent time to decide incoming packet priority.

Q. What is the running time of PQ-SORT with such a priority queue?

We obtain an algorithm that is more traditionally known as INSERTION-SORT.

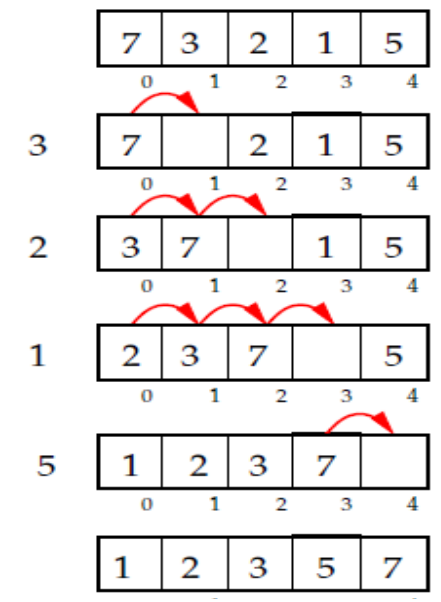

Figure 1: An example execution of INSERTION-SORT.

A direct, in-place, implementation of INSERTION-SORT:

Figure 8. An example execution algorithm in PQ algorithm

As steps execution that has been shown in Figure 8, can be simplified into flowchart as depicted in Figure 9.a while Figure 9.b is showing algorithm in PQ which using an insertion-sort. In Figure 9.a there are four decisions and five process of steps execution while in Figure 9.b there are 14 decisions and five process of steps execution. So, based on Figure 8, and 9.b our summarized that can cause incoming packet in TCP is starvation. 


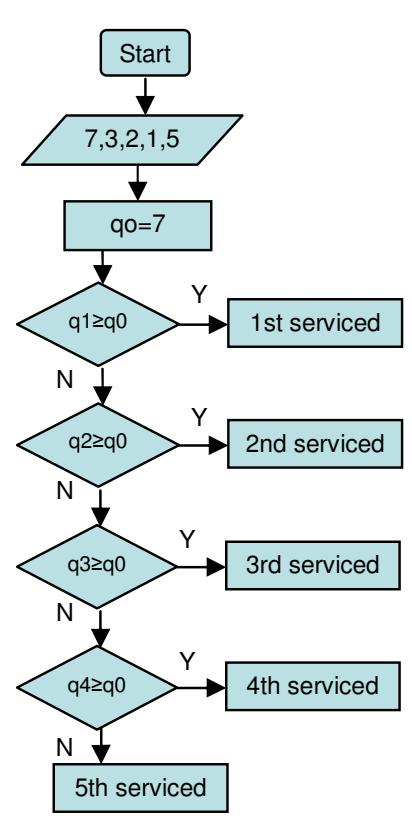

(a)

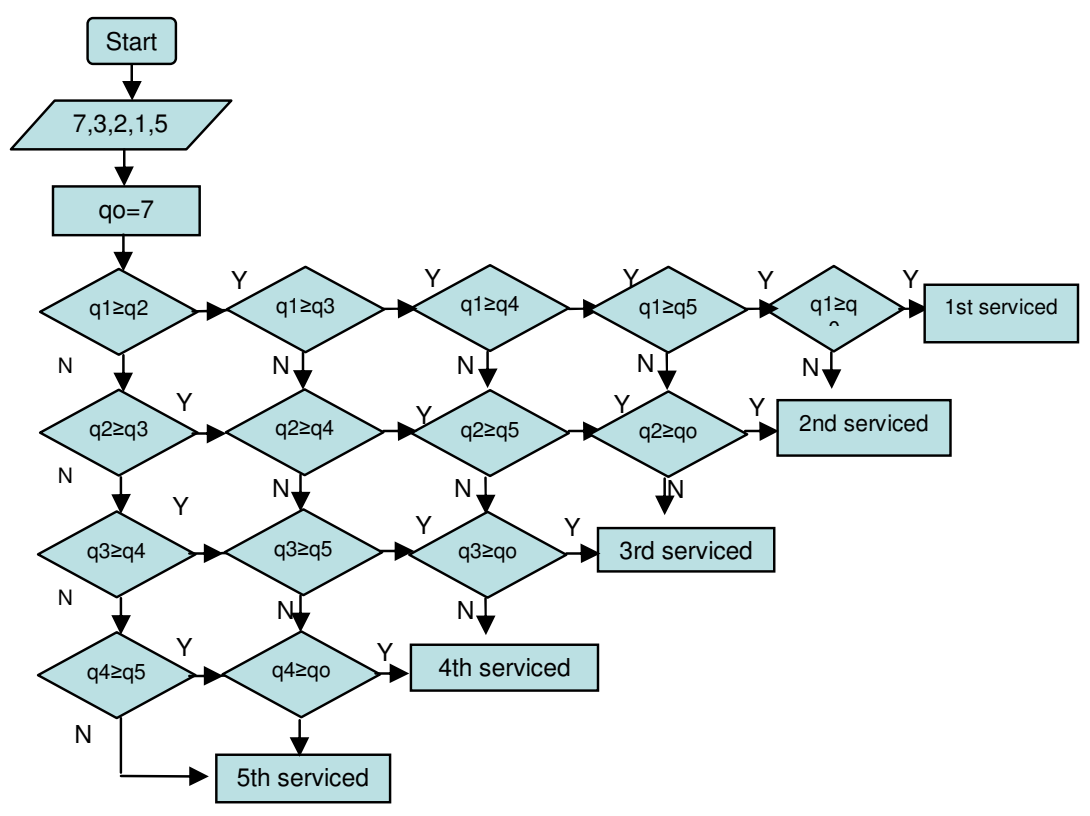

(b)

Figure 9 (a) and (b) An execution new queuing algorithm vs PQ algorithm

Starvation is happening on the PQ algorithm is caused by many recursively (repetitions) as shown in Figure 9 (b) and the algorithm flow chart in Figure 9 (b) when operating and PQ algorithm is always service the highest priority so that by simplifying the algorithm as shown in Figure 9(a) will be able to reduce recursively.

Comparison between PQ algorithm and our algorithm is where PQ classifies packets according to certain criteria in the IP network is also described part of introduction section. PQ classifies packets to up to four classes; each associated with one of four priority queues, and gives each class an appropriate packet queue. Fourth priority queue is high, medium, normal, and low queues in order of priority. This algorithm is greedy, because the incoming packet is always serviced first while second and other priority is never serviced that can cause others incoming packet is starvation. In our algorithm, fourth priority is simplified just into three priorities namely High, Medium, and Low. Incoming packet is arranged in buffering packet namely High, Medium and Low as well. Our algorithm also considering priority, arrival time and demand, while in PQ algorithm is just based on priority. The other rules in this algorithm are limited a number until three queuing incoming packets as described. 
International Journal of Network Security \& Its Applications (IJNSA), Vol.3, No.1, January 2011

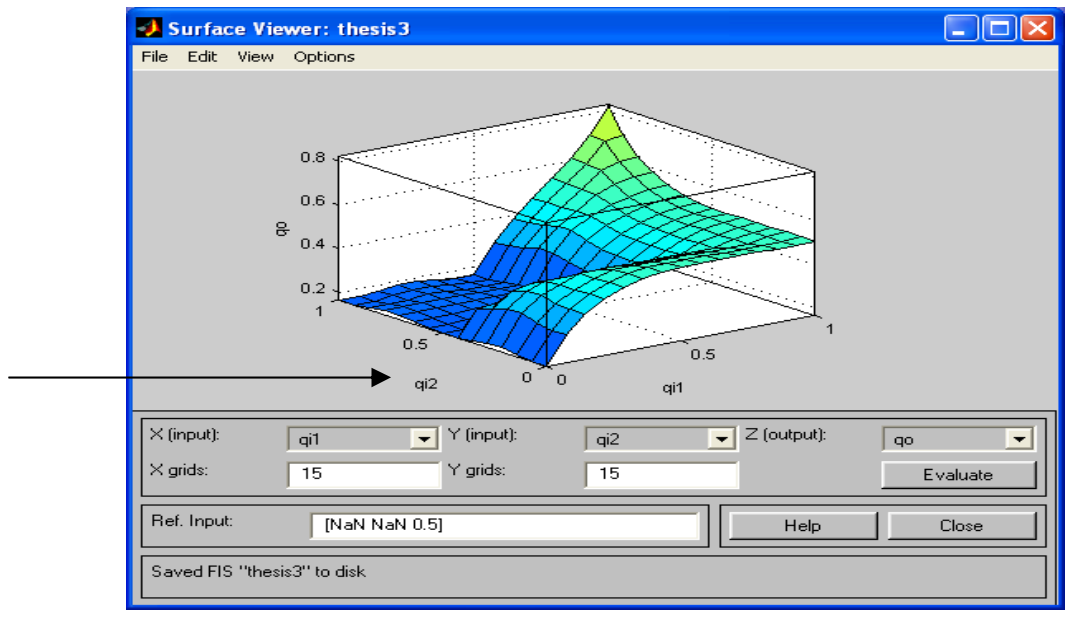

Figure 10. Fuzzy surface classifying view

Figure 10 is a Fuzzy surface view which is based on rules incoming packet. As shown in Figure 10 , that incoming packet with lower priority (as indicated by the arrow) is still serviced after next three queuing, so that all of the queuing incoming packets with lower priority in buffering packet, it can be serviced as well. So this is a purpose to reduce delay, and so that starvation is not occured for lower priority as well.

\section{CONCLUSION}

PQ algorithm is one of many queuing scheduling algorithm that can be used in VoIP network, which has investigated in this paper. A new queuing scheduling algorithm is based on PQ algorithm has been proposed to reduce delay in VoIP network. This algorithm created is based existing technique in PQ algorithm based on priorities and combined with fuzzy logic. This algorithm can classify a different packet from incoming packets and also can reduce recursive loop and starving as occurs in PQ algorithm. As shown in Figure 8., 9(a) and 9(b) that long execution in PQ algorithm can cause lower priority is starvation. This long execution algorithm affects to delay of incoming packets in TCP protocol. Further work, this paper continues on buffering and scheduling phase that would be extended to next paper.

\section{ACKNOWLEDGEMENTS}

This research is supported by DIKTI (High Education) Jakarta Indonesian scholarship for allowing us to further our study at Universiti Teknologi Malaysia (UTM).

\section{REFERENCES}

[1] Algorithms and Data Structures Priority Queues and Simple Sorting, rev. 07/02/2009 21:55.

[2] Chinchol Kim, Sangcheol Shin, Sangyong Ha, Kyongro Yoon, and Sunyoung Han, (2004). Architecture of End-to-End QoS for VoIP Call Processing in the MPLS Network, J. Solé-Pareta et al. (Eds.): QofIS 2004, LNCS 3266, pp. 44-53, 2004.p Springer-Verlag Berlin Heidelberg 2004.

[3] David Ahlgren, (2005). Introduction to SIP and VoIP Technology and Business Development, Copyright - Ahlgren 2005 AhlTek.

[4] ERRAMPALli, Madhu, (2007). Fuzzy Logic based Microscopic Traffic Network Simulation (FloMITS) Model,Urban and Regional Planning Lab.Graduate School of Engineering Gifu University. 
International Journal of Network Security \& Its Applications (IJNSA), Vol.3, No.1, January 2011

[5] Hesham N. Elmahdy And Mohamed H. N. Taha, (2009). The Impact of Packet Size and Packet Dropping Probability on Bit Loss of VoIP Networks, Faculty of Computers and Information, Cairo University, Giza, Egypt ehesham@mailer.eun.eg, mnasrtaha@gmail.com

[6] Hyun Cheol Cho, M. Sami Fadali, Jin Woo Lee, Young Jin Lee, and Kwon Soon Lee, (2007). Lyapunov-based Fuzzy Queue Scheduling for Internet Routers, International Journal of Control, Automation, and Systems, vol. 5, no. 3, pp. 317-323, June 2007

[7] K. Kim, B. Kahng and D. Kim, (2009). Jamming transition in traffic flow under the priority queuing protocol, Department of Physics and Astronomy, Seoul National University - Seoul 151-747, South Korea E-mail: bkahng@snu.ac.kr Received 11 January 2009, accepted for publication 14 May 2009 Published 16 June 2009

[8] Lars Arge, (2007). I/O-Efficient Algorithms and Data Structures, Madalgo Center For Massive Data Algorithmic, University of AarhusMay 28-29, 2007

[9] Leila Boukhalfa, Pascale Minet, Serge Midonnet, (2008). A Solution for QoS Support in Wireless Ad hoc Networks , International Journal On Advances in Networks and Services, vol 1 no 1, year 2008, http://www.iariajournals.org/networks_and_services/

[10] Martin J. Fischer, Denise M. Bevilacqua Masi, and Patrick V. McGregor, (2007). Efficient Integrated Services in an Enterprise Network, Published by the IEEE Computer Society 1520-9202/07/\$25.00 () 2007 IEEE.

[11] Miroslav Vozňák, František Hromek, (2007). Analytic Model of Delay Variation Valid for RTP, CESNET technical report number.

[12] Mohammed Alkhawlani and Aladdin Ayesh, (2008). Access Network Selection Based on Fuzzy Logic and Genetic Algorithms, Faculty of Computing Sciences and Engineering, De Montfort University, Leicester LE1 9BH, UK Correspondence should be addressed to Aladdin Ayesh, aayesh@dmu.ac.uk Received 17 September 2007; Accepted 20 February 2008

[13] Mohsin Iftikhar, Tejeshwar Singh, Bjorn Landfeldt. Mine Caglar, (2008). Multiclass G/M/1 queueing system with self-similar input and non-preemptive priority a School of Information Technologies, University of Sydney, J121 Cleveland Street, Redfern, Sydney, NSW 2006, Australia Department of Mathematics, Koc University, Istanbul, Turkey Available online 3 January 2008

[14] Racha Ben Ali and Samuel Pierre, (2005). UMTS-to-IP QoS Mapping for Voice and Video Telephony Services, École Polytechnique de Montréal Yves Lemieux, Ericsson Research Canada

[15] S. Dasgupta, C. H. Papadimitriou, and U. V. Vazirani, (2006). Algorithms, Copyright 2006 July 18, 2006.

[16] Sangho Shin, Henning Schulzrinne, (2008). Towards the Quality of Service for VoIP traffic in IEEE 802.11 Wireless Networks, Department of Computer Science Columbia University, 2008.

[17] Sanjay Rungta, Omer Ben Shalom, (2006). Enterprise and Converged Network-One Network for Voice, Video, Data, and Wireless, Intel Technology Journal, Volume 10, Issue 1, 2006.

[18] T.Ferrari, A.Ghiselli, C.Vistoli, F.Callegati, G.Corazza, J. Mongay, G.Pau, C.Raffaelli, (1999), A QoS Measurement-Based Analysis of the Expedited Forwarding Per Hop Behaviour, Istituto Nazionale di Fisica Nucleare (INFN-CNAF)

[19] Transition network, (2003). Quality of Service (QoS) in High-Priority Applications, USA Headquarters 6475 City West Parkway Minneapolis, MN 55344 USA tel 952.941 .7600 toll free 800.526.9267 fax 952.941.2322 info@transition.com www.transition.com

[20] Wei Wang, Soung C. Liew, and Victor O. K. Li, (2004). Solutions to Performance Problems in VoIP over 802.11 Wireless LAN, Department of Information Engineering and Department of Electrical and Electronic Engineering The University of Hong Kong

[21] Yaning Wang, Linghang Fan, Dan He, Rahim Tafazolli, (2008). Performance comparison of scheduling algorithms in network mobility environment, Centre for Communication System Research, 
International Journal of Network Security \& Its Applications (IJNSA), Vol.3, No.1, January 2011

University of Surrey, Guildford, UK Received 22 February 2007; received in revised form 6 November 2007; accepted 6 November 2007 Available online 4 December 2007.

[22]Zadeh, L. A. (1987). Fuzzy sets and applications: Selected papers by L.A. Zadeh. New York: Wiley.

\section{Authors}

Short Biography

Suardinata, he is received the Diploma III 1999 in Information Management at AMIK Riau, Indonesia, Bachelor Degree in Information Engineering from STMIK Riau, Indonesia, and Master Degree in Information Technology from Universitas Putra Indonesia, Padang, Indonesia, Currently he is a Ph.D. student in the Dept. of Computer System and Communication, Faculty of Computer Science and Information System, University Teknologi Malaysia, Johor Bahru Malaysia. He has been working as Lecturer at STMIK Indonesia Padang from 2005 in the Dept. of Computer Science and Information Systems, STMIK Indonesia Padang. His research interests include Multimedia and Voice over IP network, Network Security, Traffic Engineering and Quality of Service issues in IP networks, Wireless Ad-Hoc Networks, and Distributed Systems.

Kamalrulnizam bin Abu Bakar obtained his Ph.D degree in Computer Science (Network Security) from Aston University (Birmingham, UK) in 2004, B.S 1996 in Computer Science, Universiti Teknologi Malaysia and M.S. in Computer Communication \& Networks, Leeds Metropolitan University, UK. in 1998. Currently, he is an Associate Professor in Computer Science at Universiti Teknologi Malaysia (Malaysia) and member of the "Pervasive Computing" research group. He involves in several research projects and is the referee for many scientific journals and conferences. His specialization includes mobile and wireless computing, information security and grid computing.

Nimitr Suanmali, is a Ph.D. student in the Dept. of Computer System and Communication, Faculty of Computer Science and Information System, University Teknologi Malaysia, Johor Bahru Malaysia. He received his B.Sc. degree in computer science from Suan Dusit Rajabhat University,Thailand in 1998, M.Sc. degree in Information Technology from King Mongkut's University of Technology Thonburi, Thailand in 2003. Since 2003, he has been working as lecturer at Suan Dusit Rajabhat University, Bangkok Thailand. His research interests include Network Security, Intrusion Detection and Intrusion Prevention, Wireless Ad-Hoc Networks, and Distributed

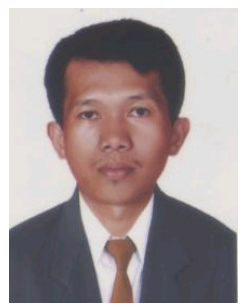
Systems.
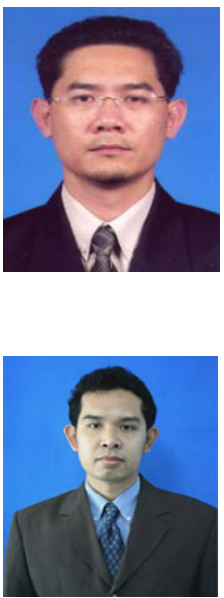